\title{
Mesenchymal stem cells transplanted into spinal cord injury adopt immune cell-like characteristics
}

\author{
Ramil Hakim 1,3,5, Ruxandra Covacu ${ }^{1,2,3}$, Vasilios Zachariadis ${ }^{4}$, Arvid Frostell ${ }^{3,5,6}$, Sreenivasa Raghavan Sankavaram²,3, \\ Lou Brundin ${ }^{1,3,5^{*}+}$ (iD and Mikael Svensson ${ }^{3,5,6+}$
}

\begin{abstract}
Background: Mesenchymal stem cells (MSCs) and their cellular response to various stimuli have been characterized in great detail in culture conditions. In contrast, the cellular response of MSCs in an in vivo setting is still uncharted territory. In this study, we investigated the cellular response of MSCs following transplantation into spinal cord injury (SCI).

Methods: Mouse bone marrow-derived MSCs were transplanted $24 \mathrm{~h}$ following severe contusion SCl in mice. As controls, MSCs transplanted to the uninjured spinal cord and non-transplanted MSCs were used. At 7 days post transplantation, the MSCs were isolated from the $\mathrm{SCl}$, and their global transcriptional changes, survival, differentiation, proliferation, apoptosis, and phenotypes were investigated using RNA sequencing, immunohistochemistry, and flow cytometry.
\end{abstract}

Results: MSCS transplanted into SCI downregulated genes related to cell-cycle regulation/progression, DNA metabolic/biosynthetic process, and DNA repair and upregulated genes related to immune system response, cytokine production/response, response to stress/stimuli, signal transduction and signaling pathways, apoptosis, and phagocytosis/endocytosis. MSCs maintained their surface expression of Sca1 and CD29 but upregulated expression of CD45 following transplantation. Transplanted MSCs maintained their surface expression of MHC-I but upregulated surface expression of MHC-II. Transplanted MSCs survived and proliferated to a low extent, did not express Caspase-3, and did not differentiate into neurons or astrocytes.

Conclusion: MSCS transplanted into SCl upregulate expression of CD45 and MHC-II and expression of genes related to cytokine production, phagocytosis/endocytosis, and immune cells/response and thereby adopt immune cell-like characteristics within the recipient.

Keywords: Mesenchymal stem cells, Spinal cord injury, Cellular response, Transcriptional changes, Phenotypes, Differentiation, Proliferation, Graft survival

\section{Background}

Traumatic spinal cord injury (SCI) leaves an affected individual with reduced motor, sensory, and autonomic functions as well as reduced quality of life. Mesenchymal stem cells (MSCs) are fibroblast-like cells that adhere to plastic,

\footnotetext{
* Correspondence: lou.brundin@ki.se

${ }^{\dagger}$ Lou Brundin and Mikael Svensson contributed equally to this work.

'Department of Neurology, Karolinska University Hospital, 17176 Stockholm, Sweden

${ }^{3}$ Department of Clinical Neuroscience, Karolinska Institutet, 17176 Stockholm, Sweden

Full list of author information is available at the end of the article
}

grow in colonies, self-renew, and differentiate along the mesodermal lineage. MSCs transplanted following SCI in rodents reduce glial scar formation, cystic cavity size, and lesion size and enhance angiogenesis, tissue sparing, axonal regeneration, and re-myelination. They also alternatively activate macrophages and reduce inflammation. These beneficial modulations have been correlated with improved recovery in hind limb motor function [1-8]. It has been suggested that transplanted MSCs differentiate along the ectodermal lineage $[4,6,9]$, but others have suggested different mechanisms $[2,10,11]$. 
Transplanted MSCs have demonstrated the capacity to enhance the expression of neurotrophic and growth factors in the recipient $[2,4,7]$. Both the differentiation and the elevation of neurotrophic and growth factors have been proposed as possible explanations for the enhanced recovery of hind limb motor function $[2,7,9,12,13]$. In culture conditions, MSCs release extracellular vesicles (MSC-EVs) containing proteins, trophic factors, cytokines [14, 15], mRNA $[14,16]$, and microRNA $[14,17,18]$ through which they modify neighboring cells and mediate neuroprotective and immunomodulatory effects [19-21]. The regenerative potential of MSC-EVs seems to depend on the culture conditions $[22,23]$. For the time being, the release of MSC-EVs is thought to be the main mechanism of action of MSCs.

The cellular response of MSCs in culture and the effect of MSC transplantation following SCI have been reported repeatedly. However, the SCI environment is vastly more complex than what can be simulated in culture conditions, and observations made concerning the cellular response of MSCs in culture conditions cannot be assumed to valid in an in vivo environment. Thus, in this study, we investigated the cellular response of transplanted MSCs following SCI with regard to their transcriptional changes, phenotypic profile, proliferation, apoptosis, and differentiation. Understanding the cellular response and especially the mechanism of action of MSCs allows for modification, enhancement, or replacement of MSCs, which could ultimately be transformed into a cell-free therapy that maximizes the patient's recovery following SCI.

\section{Methods}

\section{Mice}

Wild-type female mice (C57BL/6J, 10-12 weeks, 18-20 g) were purchased from Scanbur (Stockholm, Sweden). Animals were kept at $20^{\circ} \mathrm{C} \pm 1{ }^{\circ} \mathrm{C}$ in a room equipped with a 12-h:12-h light/dark cycle switch. Animal care and experiments were approved by the local ethical committee (Stockholm, Sweden) and maintained according to permits (N196/15, N12317-2017, N38/16) and local guidelines at Karolinska Institutet.

\section{Contusion spinal cord injury}

Animals were anesthetized using $0.5 \mathrm{mg} / \mathrm{kg}$ medetomidine i.p. (Domitor ${ }^{\circ}$ vet., Orion Pharma Animal Health, 1 $\mathrm{mg} / \mathrm{ml}$ ) and $75 \mathrm{mg} / \mathrm{kg}$ ketamine i.p. (Ketador vet., Salfarm Scandinavia, $100 \mathrm{mg} / \mathrm{ml}$ ) in combination with $0.05 \mathrm{mg} / \mathrm{kg}$ buprenorphine s.c. (Temgesic ${ }^{\circ}$, Indivior 0.3 $\mathrm{mg} / \mathrm{ml}$ ) and $5 \mathrm{mg} / \mathrm{kg}$ carprofen s.c. (Rimadyl ${ }^{\circ}$ vet. Orion Pharma Animal Health, $50 \mathrm{mg} / \mathrm{ml}$ ). Animals were given $0.1 \mathrm{ml}$ normal saline s.c., weighed, and shaved on the back. The skin was incised, and the paravertebral muscles separated. The vertebrae at Th10 were stabilized using bilateral fixators in a stereotaxic frame (Model 900 \& 900-c, Kopf ${ }^{\circ}$ ). Using a surgical drill (Anspach ${ }^{\circ}$, $\mathrm{EMAX}^{\circ}$ 2), the dorsal part of the vertebrae was removed. The dura mater was cut open. Using the infinite horizon impactor (Infinite Horizon, IH-0400), a severe contusion spinal cord injury (SCI, $75 \mathrm{kdyn}$ ) was induced at Th10. When no hemorrhage was visible in the injury area, bupivacaine (Marcain ${ }^{\circ}$, Aspen Nordic, $2.5 \mathrm{mg} / \mathrm{ml}$ ) was injected into the surrounding tissue, and the skin was sutured (Vicryl, 4.0). Buprenorphine $0.05 \mathrm{mg} / \mathrm{kg}$ was administrated twice daily and carprofen $5 \mathrm{mg} / \mathrm{kg}$ once daily for a total of 3 days post-surgery. Mice were weighed weekly, and $25 \%$ weight loss was deemed the humane endpoint. The urine bladders were manually compressed until recovery of reflexive bladder emptying.

\section{Establishment of mesenchymal stem cells}

Bone marrow mouse mesenchymal stem cells (MSCs) were established from 4-6-week-old male C57BL/6J mice using methods described elsewhere [24]. Briefly, animals were sacrificed, and the tibia and femur from both legs were harvested. The epiphysis of the tibias and femurs were cut open, and the bone marrow was extruded by flushing with pre-warmed basal medium (89\% $\alpha$-MEM (Gibco , 22561054), 1\% Pen-Strep (Gibco ${ }^{\circ}$, $10.000 \mathrm{U} / \mathrm{ml}, 15140122)$, and $10 \%$ fetal bovine serum $\left.\left(\mathrm{Gibco}^{\circ}, 10082147\right)\right)$. The bone marrow was dissociated by trituration and filtered through a 70- $\mu \mathrm{m}$ cell strainer (Corning, Inc., 352350). Bone marrow cells were re-suspended in basal medium and plated at a density of $1.45 \times 10^{6} \mathrm{cells} / \mathrm{cm}^{2}$ in $100-\mathrm{mm}$ tissue culture-treated dishes $\left(150350\right.$, $\left.\mathrm{Nunc}^{\mathrm{rn}}\right)$. At $8-10$ days post initial plating, the plastic adherent bone marrow cells were harvested using $0.5 \%$ trypsin $\left(\mathrm{Gibco}^{\mathrm{mm}}, 15400054\right)$ for immune depletion. MSCs were depleted of CD34-positive (eBioscience, 13-0341-85), CD45-positive (BD, 553078), and CD11bpositive (BD, 553309) cells. Immune-depleted MSCs were characterized in terms of differentiation potential, phenotypic profile, colony-forming potential, and growth dynamics.

\section{Transfection of mesenchymal stem cells}

A modified enhanced episomal vector system (EEV; CAT\#EEV600A-1, System Biosciences) was used to mark the MSC genetically with mCherry. A construct containing the mCherry cDNA linked to the cDNA of the HBEGF/DTR $[25,26]$ via a 2 a self-cleaving peptide linker was directionally cloned into the multiple cloning sites of the vector, downstream of the CAG promoter. Prior to transfection, the plasmid was cleared from endotoxins by using the endo-free kit (Qiagen, 12362). At $96 \mathrm{~h}$ prior to transplantation, MSCs were plated at a density of $10 \mathrm{e} 3 \mathrm{cells} / \mathrm{cm}^{2}$ in tissue culture-treated 
six-well plates (Corning ${ }^{\mathrm{Tw}}, 3516$ ). At $48 \mathrm{~h}$ prior to transplantation, the MSCs were transfected $(2.5 \mu \mathrm{l} \mathrm{cDNA} /$ well) using Lipofectamine ${ }^{\text {Tx }} 3000$ (Invitrogen, L3000001). The transfection efficiency was 40-50\% (Additional file 1: Figure S1A-C).

\section{Transplantation of mesenchymal stem cells}

Prior to transplantation ( $24 \mathrm{~h}$ post SCI, $48 \mathrm{~h}$ post transfection), the fluorescence (mCherry) of the MSCs was confirmed (Zeiss, Axiovert 200). The MSCs were harvested using $0.5 \%$ trypsin, washed and collected in 0.2-ml Eppendorf tubes, and kept at $4{ }^{\circ} \mathrm{C}$ until transplantation. Animals were anesthetized using the same principles as during SCI induction, the sutures were opened, and the spinal cord was exposed. Using a glass capillary needle (WPI, 1B150F-6) attached to a 10- $\mu$ l syringe (Hamilton $\left.{ }^{\circ}, 80330\right), \sim 0.5 \times 10^{6}$ MSCs were injected into the SCI epicenter under microscopic visualization. The skin was sutured (Vicryl, 4.0), and post-operative procedures as described for SCI were implemented.

\section{Sacrifice and tissue harvesting}

At 7 days post transplantation, animals were euthanized using pentobarbitalnatrium. For histological evaluation, animals were sacrificed at 7 and 14 days post transplantation. Animals were transcardially perfused with $1 \times \mathrm{PBS}$ using a peristaltic pump (Watson Marlow, 120S). Spinal cords intended for evaluation using flow cytometry of fluorescence-activated cell sorting (FACS) were dissected and stored in $1 \times \mathrm{PBS}$ on ice. Animals intended for histological evaluation were further perfused with paraformaldehyde (PFA, 4\%). These spinal cords were dissected and post-fixated in PFA overnight.

\section{Isolation of transplanted mesenchymal stem cells from the spinal cord}

Spinal cords were dissociated using trituration in L-15 medium (Thermo Fisher, 11415064) containing $10 \mathrm{U} / \mathrm{ml}$ papain (Worthington, L5003126). Two hundred units per milliliter DNAse (Sigma, D7291) was added during the dissociation. Papain was inactivated using $1 \%$ bovine serum albumin (BSA; Gibco ${ }^{\circ}$ 15260-037) in $1 \times$ DPBS. Myelin was removed using a 30\% Percoll (Sigma, P1644) gradient and centrifugation at $750 \times g$ for $10 \mathrm{~min}$ at $4{ }^{\circ} \mathrm{C}$ with slow brake. The pellet was re-suspended in FACS buffer (1\% BSA, 2 mM EDTA (Gibco ${ }^{\circ}$ 15575-038), 25 mM HEPES (Sigma, H0887)) and filtered through a pre-wet $100-\mu \mathrm{m}$ cell strainer (Corning, 431752) followed by a pre-wet $40-\mu \mathrm{m}$ strainer (Corning, 431750). mCherry+MSCs (Additional file 2: Figure S2A-R) were isolated from the dissociated spinal cord using FACS (BD Influx $\left.{ }^{\mathrm{nt}}\right)$. Sorted cells $(28,000 \pm 14,000 \mathrm{MSCs})$ were collected in FACS buffer, centrifuged at $300 \times g$ for $5 \mathrm{~min}$, and re-suspended in $1 \mathrm{ml}$ Trizol reagent (Thermo Fisher,
15596026), incubated for $5 \mathrm{~min}$, vortexed, frozen on dry ice, and stored at $-70{ }^{\circ} \mathrm{C}$ until downstream processing.

\section{Extraction of RNA from isolated mesenchymal stem cells} RNA from isolated MSCs was isolated using Trizol (manufacturer's protocol). Contaminating genomic DNA was removed during the RNA isolation by on-column digestion with DNAse (DNAse I Qiagen, 79254). RNA clean-up was conducted using the RNeasy micro kit (Qiagen, 74004). RNA was stored at $-70^{\circ} \mathrm{C}$ until sequencing.

\section{Analysis of global transcriptional changes in mesenchymal stem cells}

Sequencing libraries were prepared using the SMARTer Stranded Total RNA-Seq Kit - Pico Input Mammalian kit (Clontech). Libraries were sequenced $2 \times 125 \mathrm{bp}$ in two lanes using the HiSeq2500 system and v4 sequencing chemistry (Illumina Inc.) to a combined total of at least $15.7 \times 10^{6}$ reads/sample. TrimGalore (Babraham Bioinformatics) was used for the removal of adapter sequences and low-quality regions. The splice-aware aligner STAR was used for aligning remaining pair-end reads to the mouse genome (build GRCm38). FeatureCounts and Ensembl annotation (release 81) were used for summarization of read counts over genes. Annotation and data analysis were conducted in $\mathrm{R}$ (version 3.5.1) using packages limma and edgeR with annotations from Mus.musculus (https://www.bioconductor.org/), GEO accession number: GSE125176.

\section{Functional analysis}

Significantly differentially expressed genes (FDR $<0.01$, $\operatorname{LogFC}=1$ ) for each contrast were analyzed using over-representation enrichment analysis (ORA) and network topology-based analysis (NTA) using WEB-based Gene SeT AnaLysis Toolkit (WebGestalt) implemented with R package "WebGestaltR." Up- and downregulated genes in each contrast were analyzed separately. In ORA, the Gene Ontology (GO) terms related to biological process (BP), molecular function (MF), and cellular component $(\mathrm{CC})$ were investigated. Furthermore, in ORA, pathways were investigated using Kyoto Encyclopedia of Genes and Genomes (KEGG) terms. In NTA, both network retrieval and prioritization (NRP) and network expansion (NE) were used for network construction. All terms (and related genes) which fulfilled FDR $<0.01$ were exported for each method (BP, MF, CC, KEGG, NRP, NE), contrast and direction. Each term was then manually categorized into more general categories for enhanced interpretation. For each contrast and category, the median FDR was calculated. Competitive gene set testing accounting for inter-gene correlation was performed on all unique genes in each category for each contrast. A category was deemed significant if median 
FDR for GO/KEGG terms and exact FDR for competitive gene set testing were both $<0.05$ for that specific category. The categories were ordered based on the number of GO/KEGG terms that were detected for the specific category. Gene set enrichment analysis (GSEA) was conducted using Molecular Signatures Database (MSigDB, v6.2) using collections: hallmark gene sets, curated gene sets $(\mathrm{C} 2)$, and immunologic gene sets $(\mathrm{C} 7)$.

\section{Flow cytometry}

Spinal cords containing transplanted MSCs were dissociated using papain (above). Cells were blocked in Mouse Fc Block (BD, 553141) for $5 \mathrm{~min}$ and stained using pre-conjugated antibodies (Table 1) in 100- $\mu$ l FACS buffer on ice for $30 \mathrm{~min}$. Following the wash, the cells were re-suspended in $250 \mu \mathrm{l}$ FACS buffer. Non-transplanted MSCs were harvested (above) from culture $48 \mathrm{~h}$ post transfection and stained in the same fashion. Flow cytometry was conducted using a BD LSRFortessa ${ }^{\text {TM }}$ cell analyzer and data analyzed in Kaluza Analysis Software (Beckman Coulter).

\section{Immunohistochemistry}

Post-fixed spinal cords were cryo-protected in 15\% and $30 \%$ sucrose (Sigma, S9378) in $1 \times$ PBS. Spinal cords were mounted in cryomolds (Tissue-Tek ${ }^{\circ}$ Cryomold $^{\circ}$, 420572) using compound (Tissue-Tek ${ }^{\circ}$ O.C.T..$^{\mathrm{mu}}$ ) and rapidly frozen to $-60^{\circ} \mathrm{C}$. Twenty-micrometer coronal sections were produced using a cryostat (Leica, CM1850, $-22^{\circ} \mathrm{C}$ ) and mounted on slides (VWR, SuperFrost ${ }^{\circ}$ Plus, 48311-703). Sections were thawed, rehydrated in $1 \times$ PBS, blocked for $2 \mathrm{~h}$ at $\mathrm{RT}$ in blocking solution $(0.3 \%$ Triton X-100 (Sigma, 93443), 5\% normal goat serum (Serotec, 301104, $1 \times$ PBS and $0.01 \%$ sodium azide (Sigma, S-2002)). Primary antibody (Table 1) was added, and sections were incubated at $4{ }^{\circ} \mathrm{C}$ for $24 \mathrm{~h}$. Sections were rinsed in $1 \times \mathrm{PBS}$ followed by incubation in secondary antibody (Table 1) at RT for $1 \mathrm{~h}$. Sections were incubated at RT for 20 min with nucleic acid stain (Hoechst 33258, Invitrogen $^{\text {TM }}$ H3569). Prior to confocal microscopy, the slides were rinsed and mounted using Mowiol (Sigma, 81381) and a cover slip (Marienfeld, 010243).

\section{Immunocytochemistry}

FACS isolated MSCs were collected at $500 \times g$ for $5 \mathrm{~min}$ at $4{ }^{\circ} \mathrm{C}$ and re-suspended in basal medium. Cells were plated at a density of 20.000 cells $/ \mathrm{cm}^{2}$ on tissue culture-coated slides (Nunc $^{\text {tm }}$ Lab-Tek $^{\circ}$ Chamber Slide ${ }^{\mathrm{ma}}$, 177402) in $0.3-\mathrm{ml}$ basal medium. Following 3 days of culture, the MSCs were washed and fixated using 2\% PFA for $30 \mathrm{~min}$. MSCs were stained using the procedure described for frozen sections and imaged using confocal microscopy.

\section{Proliferation assay}

MSCs in culture were exposed to 1:1000 5-ethynyl2 '-deoxyuridine (EdU; Thermo Fisher, A10044) for $24 \mathrm{~h}$ prior to assessment of proliferation. Proliferation of transplanted MSCs was assessed by administration of $0.75 \mathrm{mg} / \mathrm{ml} \mathrm{EdU} \mathrm{in} \mathrm{the} \mathrm{drinking} \mathrm{water} \mathrm{of} \mathrm{the} \mathrm{mice.}$ Drinking water containing EdU was offered to the animals from transplantation to sacrifice. The water was supplemented with 1\% sucrose (Sigma Aldrich, S0389). Proliferation of MSCs in culture was assessed using flow cytometry (above). Proliferation of transplanted MSCs was assessed using immunohistochemistry. For both evaluations, the Click-iT ${ }^{\mathrm{mm}}$ Plus EdU Alexa Fluor ${ }^{\mathrm{rm}}$ 488/ 555 Imaging Kit (Thermo Fisher, C10637) was used according to the manufacturer's instructions.

\section{Differentiation assay}

Differentiation of transplanted MSCs was evaluated using immunohistochemistry. The co-expression of mCherry and either GFAP, NeuN, or TuJ1 (Table 1) was evaluated using confocal microscopy.

Table 1 Primary, secondary, and pre-conjugated antibodies

\begin{tabular}{|c|c|c|c|c|c|}
\hline Antibody type & Host & Target & Fluorochrome & Manufacturer (ID) & Dilution \\
\hline \multirow[t]{5}{*}{ Primary } & Mouse & NeuN & NA & Merck Millipore (MAB377) & $1: 1000$ \\
\hline & Mouse & TuJ1 & NA & Merck Millipore (MAB1637) & $1: 100$ \\
\hline & Rabbit & GFAP & NA & Dako (Z0334) & $1: 1000$ \\
\hline & Rat & MHC-II & NA & Abcam (ab25333) & $1: 200$ \\
\hline & Rabbit & Caspase-3 & NA & Abcam (ab13847) & $1: 100$ \\
\hline \multirow[t]{2}{*}{ Secondary } & Goat & Mouse & Alexa 488 & Life Technologies (A11001) & $1: 500$ \\
\hline & Goat & Rabbit & Alexa 488 & Life Technologies (A11008) & $1: 400$ \\
\hline \multirow[t]{5}{*}{ Pre-conjugated } & NA & CD29 & APC & eBioscience (17-0291-80) & $1 \mu \mathrm{g}$ per $1 \times 10^{6}$ cells \\
\hline & NA & Sca1 & FITC & BD (561077) & \\
\hline & NA & CD45 & v450 & BD (560697) & \\
\hline & NA & MHC-I & FITC & BioLegend (125508) & \\
\hline & NA & MHC-\|I & FITC & BioLegend (107606) & \\
\hline
\end{tabular}


Confocal microscopy and automatic cell quantification Stained frozen sections and stained fixed MSCs were imaged using a confocal microscope (Zeiss LSM 880 Airyscan). For estimation of proliferation, differentiation, apoptosis, and survival of mCherry+MSCs, all cells in four random sections from every animal were imaged for analysis. Orthogonal projections using $\times 40$ as well as overview images using $\times 20$ for quantification were collected. The proportion of MSCs co-expressing mCherry and the specific marker was estimated using a custom built automatic cell quantification macro (https://github.com/S-Blab/confocal_image_quantifier) implemented in Image Processing and Analysis in Java (ImageJ, 64-bit Java 1.6.0_24). For each animal and staining, the images were imported, and an image sequence was created. The macro allows the user to select one, two, or three channels for evaluation of co-localization. The macro splits the image into its three color channels. The selected color channels are multiplied, and the image is converted to binary format and water-shedded. Co-localized cells are then identified and labeled based on user-defined settings for desired size and circularity of cells. The accuracy was always evaluated using overlays. In case the macro had too low accuracy in a given image sequence, a manual cell count was done instead using the "Cell Counter" plugin in ImageJ.

\section{Statistical analysis}

Mean with 95\% confidence interval or median with range (25th and 75th percentile) was presented when appropriate. $p$ values $<0.05$ were considered significant. When applicable, the assumption of normality of the data was evaluated using Shapiro-Wilk's test. The assumption of homogeneity of variances between groups was evaluated using Bartlett's test when data was normally distributed and Fligner-Killeen's test when data departed from normality. Depending on the fulfillment of assumptions, multiple group comparisons were evaluated using Kruskal-Wallis $H$ test followed by pairwise comparisons between group levels with correction for multiple testing using Holm's method or using one-way ANOVA followed by Tukey's post hoc test. Independent two-group comparisons were conducted using the Wilcoxon rank-sum test or Student's $t$ test depending on the fulfillment of assumptions. Analysis was conducted, and figures were prepared in $\mathrm{R}$ (version 3.5.1) mainly using packages data.table and ggplot2.

\section{Results}

MSCs transplanted into $\mathrm{SCl}$ downregulate genes related to cell-cycle and DNA metabolic/biosynthetic processes and upregulate genes related to immune system response, cytokine production, and phagocytosis/endocytosis

MSCs transfected to express mCherry were transplanted into the uninjured and injured spinal cord. At 7 days following transplantation, the MSCs were isolated using
FACS. MSCs transplanted to spinal cord injury (SCI) were compared to MSCs transplanted into the uninjured spinal cord and to non-transplanted MSCs in terms of global transcriptional changes (Fig. 1a). Dimensionality reduction (Fig. 1b, Additional file 3: Figure S3A) and clustering (Fig. 1c, Additional file 3: Figure S3B, C) revealed a distinct separation between transplanted (MSC[SCI], MSC[Naive]) and non-transplanted MSCs (MSC[In vitro]). A more modest separation was detected between MSC[SCI] and MSC[Naive]. Numerous significantly differentially expressed genes were detected in contrasts MSC[SCI] vs MSC[In vitro] and MSC[Naive] vs MSC[In vitro] but not in MSC[SCI] vs MSC[Naive] indicating similarity between transplanted MSCs but a dissimilarity between transplanted and non-transplanted MSCs (Fig. 1d, e). Heat map representation of all significantly differentially expressed genes confirmed that the largest differences were found between transplanted and non-transplanted MSCs and that transplanted MSCs upregulated rather than downregulated their gene expression (Fig. 1f). Functional analysis revealed that genes mainly related to cell-cycle regulation/progression, DNA metabolic/biosynthetic processes, and DNA repair were downregulated and genes related to immune system response, cytokine production/response, response to stress/stimuli, signal transduction and signaling pathways, apoptosis, and phagocytosis/endocytosis were upregulated in transplanted MSCs as compared to non-transplanted MSCs (Fig. 1g, Table 2). Immune system response was characterized by genes related to both the innate and the adaptive immune system as indicated by the GO terms: activation of innate immune response, adaptive immune response, activation of immune response, and immune system process but also the activation of macrophages (macrophage activation). Interestingly, the cytokine production was related to cytokines commonly produced by macrophages and were mainly pro-inflammatory as indicated by the GO terms: interleukin-6 production, tumor necrosis factor production, interleukin-1 production, interferon-gamma production, interleukin-12 production, chemokine production, interleukin-8 production, interleukin-10 production, interleukin-2 production, and positive regulation of interleukin-17 production. Thus, the MSCs seem to adopt a secretory profile similar to classically activated macrophages. The genes related to signal transduction and signaling pathways were related to a variety of pathways as indicated by the GO terms: regulation of kinase activity, regulation of MAPK cascade, Ras protein signal transduction, Fc receptor signaling pathway, regulation of small GTPase mediated signal transduction, and Jak-STAT signaling pathway. Taken together, transplanted MSCs suppress genes related to cell-cycle activity and DNA processes/repair but upregulate genes related to 
A

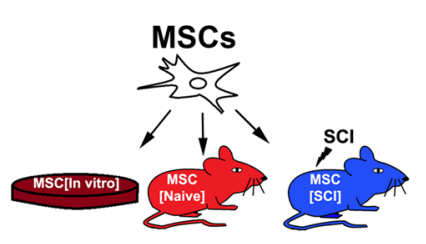

D

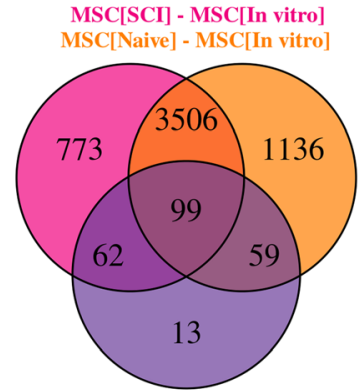

MSC[SCI] - MSC[Naive] FDR $<0.01 ; \log F C=1$
B



C

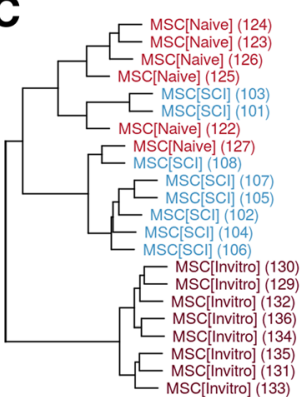

-MSC[In vitro] $(\mathrm{n}=8) \bullet M S C[N a i v e](n=6) \bullet M S C[S C I](n=8)$

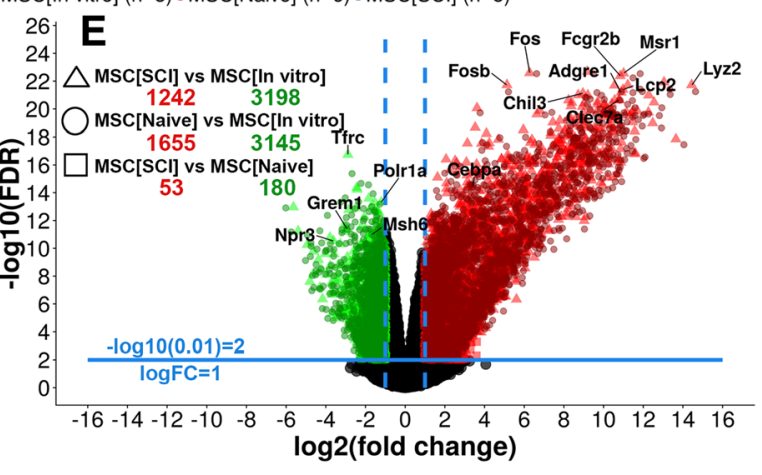

$\mathbf{F}$ 5648 differentially expressed genes (FDR $<0.01 ; \log F C=1$ )

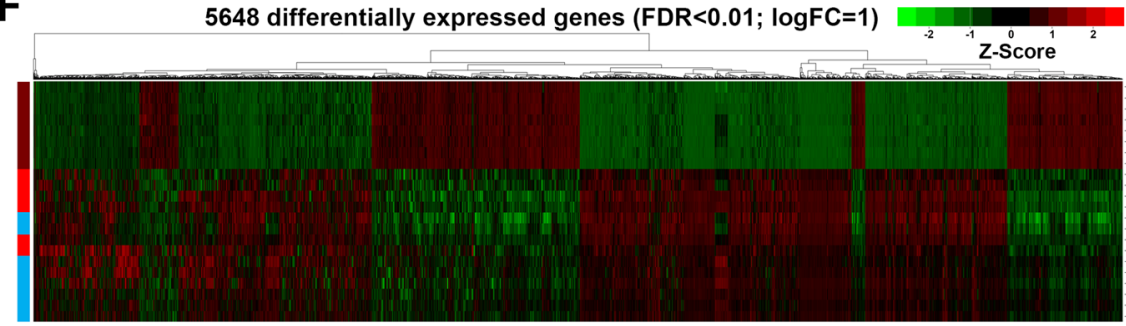

G 1. DNA Metabolic/Biosynthetic Process 9.6e-03

4. Cytokine Production \& Response

7.1e-04
Cytokine production; Cytokine secretion;
Macrophage cytokine production;

DNA geometric change; DNA packaging; regulation of DNA recombination; DNA packaging; DNA metabolic process

Tumor necrosis factor production

2. Cell Cycle Regulation \& Progression

5. Phagocytosis \& Endocytosis

\section{7e-03}

Regulation of endocytosis;

Regulation of phagocytosis;

Phagocytic vesicle; Endocytic vesicle membrane

Regulation of cell cycle;

Regulation of mitotic cell cycle

3. DNA Repair

DNA $\quad 5.1 \mathrm{e}-03$
Regulation of DNA repair;
Double-strand break repair

6. Immune System Response

Activation of innate immune response;
Adaptive immune response;
Macrophage activation;
Leukocyte mediated immunity

Fig. 1 Global transcriptional changes in MSCs. a Experimental design. Transplanted MSCs were evaluated 7 days following transplantation. b Principal component analysis using the 500 genes with the highest inter-sample variance. Spheres represent biological replicates in 3D, while squared dots represent biological replicates in $2 \mathrm{D}$ (on the walls of the box), comparing two components at a time. c Agglomerative hierarchical clustering of biological replicates presented using a dendrogram. Data is based on the value of the first and second principal component for each biological replicate. Numbers in parentheses are animal index numbers. $\mathbf{d}$ Unique number of significantly differentially expressed genes in each contrast and the number of genes shared between the contrasts. Color of text specifying the contrast corresponds to the color of the circle in the Venn diagram. e Differentially expressed genes presented using a volcano plot. $\mathbf{f}$ Heat map of unique significantly differentially expressed genes based on all three contrasts with row- and column-wise agglomerative hierarchical clustering. $\mathbf{g}$ Up- and downregulated categories for contrasts MSC[SCI] vS MSC[In vitro] and MSC[Naive] vs MSC[In vitro]. Barcode plots are presented with a $p$ value determined using a competitive gene set test. Representative Gene Ontology (biological process) terms are presented for each category. Green color indicates a category downregulated in MSC[SCI]/MSC[Naive], while red indicates a category upregulated in MSC[SCI]/MSC[Naive] as compared to MSC[In vitro] 
Table 2 Functional analysis of significantly differentially expressed genes (FDR $<0.01$; LogFC $=1$ )

\begin{tabular}{|c|c|c|c|c|c|c|}
\hline \multirow[t]{2}{*}{ Contrast } & \multirow[t]{2}{*}{ Direction } & \multirow[t]{2}{*}{ Category } & \multicolumn{2}{|c|}{ ORA and NTA } & \multicolumn{2}{|c|}{ Competitive gene set test } \\
\hline & & & Terms $(n)$ & FDR (median) & Genes $(n)$ & FDR (exact) \\
\hline \multirow[t]{30}{*}{ MSC[Naive] vs MSC[In vitro] } & \multirow[t]{4}{*}{ Down } & Cell-cycle regulation and progression & 159 & $2.1 \mathrm{e}-04$ & 276 & $2.3 e-03$ \\
\hline & & DNA recombination/metabolic process & 46 & $3.6 e-04$ & 412 & $2.9 e-03$ \\
\hline & & Chromosome (condensed) & 22 & $1.7 e-06$ & 129 & $5.5 e-03$ \\
\hline & & DNA repair and response to stress & 11 & $1.9 e-04$ & 64 & $1.9 \mathrm{e}-03$ \\
\hline & \multirow[t]{26}{*}{ Up } & Immune system response & 718 & $1.2 \mathrm{e}-05$ & 940 & $7.6 e-05$ \\
\hline & & Signaling pathway & 222 & $3.3 e-04$ & 862 & $3.7 e-06$ \\
\hline & & Cytokine production and response & 207 & 7.1e-05 & 433 & $3.4 \mathrm{e}-04$ \\
\hline & & Cellular response to stress and stimuli & 198 & $1.3 e-05$ & 1346 & $1.2 \mathrm{e}-05$ \\
\hline & & Metabolic process & 161 & $2.4 \mathrm{e}-04$ & 1345 & $2.3 e-05$ \\
\hline & & Protein modification/assembly/transport & 112 & $6.4 \mathrm{e}-05$ & 1061 & $3.4 \mathrm{e}-05$ \\
\hline & & Ion transport and homeostasis & 99 & $5.9 e-06$ & 420 & $3.4 \mathrm{e}-06$ \\
\hline & & Cell migration and chemotaxis & 91 & $8.1 e-05$ & 461 & $8.9 e-07$ \\
\hline & & Trans (cell) membrane transport & 90 & $9.0 \mathrm{e}-04$ & 372 & $9.8 e-05$ \\
\hline & & Ubiquitination and apoptosis & 83 & $2.0 \mathrm{e}-04$ & 546 & $8.8 e-05$ \\
\hline & & Signal transduction & 67 & $9.9 e-08$ & 973 & $4.5 e-06$ \\
\hline & & Tissue development and regeneration & 63 & $1.3 e-04$ & 1120 & $8.1 \mathrm{e}-06$ \\
\hline & & Biosynthetic process & 61 & $1.2 \mathrm{e}-03$ & 656 & $8.5 e-04$ \\
\hline & & Cell-cell adhesion & 59 & $1.1 e-05$ & 625 & $3.7 e-06$ \\
\hline & & Phagocytosis and endocytosis & 43 & $1.7 e-05$ & 280 & $1.0 \mathrm{e}-03$ \\
\hline & & Catabolic process & 37 & 1.0e-03 & 277 & 4.1e-04 \\
\hline & & Regulation of (cell) proliferation & 37 & $1.8 \mathrm{e}-05$ & 524 & $1.7 e-04$ \\
\hline & & Cell membrane activity & 31 & $4.0 \mathrm{e}-07$ & 791 & $2.9 e-05$ \\
\hline & & Regulation of (cell) differentiation & 28 & $7.4 \mathrm{e}-04$ & 592 & $7.5 e-07$ \\
\hline & & Synaptic function and activity & 22 & $3.1 e-03$ & 184 & $7.1 \mathrm{e}-04$ \\
\hline & & Cell and tissue morphology & 17 & $1.2 \mathrm{e}-03$ & 496 & $5.6 e-04$ \\
\hline & & Receptor binding & 15 & $1.3 e-03$ & 10 & $6.8 e-03$ \\
\hline & & Transcriptional activity & 15 & $2.8 e-03$ & 485 & $1.2 \mathrm{e}-02$ \\
\hline & & I-kappaB kinase/NF-kappaB signaling & 14 & 2.6e-04 & 82 & $2.3 e-03$ \\
\hline & & Regulation of (cell) activation & 12 & $0.0 \mathrm{e}+00$ & 267 & $1.4 \mathrm{e}-03$ \\
\hline & & Regulation of cell homeostasis & 11 & $1.5 e-03$ & 161 & $9.1 \mathrm{e}-04$ \\
\hline \multirow[t]{13}{*}{ MSC[SCI] vs MSC[In vitro] } & \multirow[t]{5}{*}{ Down } & Cell-cycle regulation and progression & 220 & $1.3 e-04$ & 253 & $3.8 e-03$ \\
\hline & & DNA recombination/metabolic process & 51 & $2.2 \mathrm{e}-04$ & 370 & $9.6 e-03$ \\
\hline & & Chromosome (condensed) & 25 & $6.1 \mathrm{e}-08$ & 122 & $8.9 e-03$ \\
\hline & & DNA repair and response to stress & 16 & $1.2 \mathrm{e}-05$ & 76 & $5.1 e-03$ \\
\hline & & Meiotic cell-cycle process & 15 & $7.5 e-05$ & 49 & $1.1 \mathrm{e}-02$ \\
\hline & \multirow[t]{8}{*}{ Up } & Immune system response & 642 & $2.5 e-05$ & 836 & $2.6 e-04$ \\
\hline & & Cellular response to stress and stimuli & 176 & $1.7 e-05$ & 1215 & $5.9 e-05$ \\
\hline & & Signaling pathway & 173 & $3.5 e-04$ & 697 & $4.9 e-05$ \\
\hline & & Cytokine production and response & 157 & $6.5 e-05$ & 399 & $7.1 e-04$ \\
\hline & & Metabolic process & 122 & 7.0e-04 & 1126 & $2.0 \mathrm{e}-04$ \\
\hline & & Ion transport and homeostasis & 117 & $4.2 \mathrm{e}-07$ & 439 & $1.4 \mathrm{e}-05$ \\
\hline & & Protein modification/assembly/transport & 102 & $3.2 \mathrm{e}-04$ & 765 & $1.2 \mathrm{e}-04$ \\
\hline & & Trans (cell) membrane transport & 81 & $4.0 \mathrm{e}-04$ & 372 & $1.4 \mathrm{e}-04$ \\
\hline
\end{tabular}


Table 2 Functional analysis of significantly differentially expressed genes (FDR $<0.01$; LogFC $=1$ ) (Continued)

\begin{tabular}{|c|c|c|c|c|c|c|}
\hline \multirow[t]{2}{*}{ Contrast } & \multirow[t]{2}{*}{ Direction } & \multirow[t]{2}{*}{ Category } & \multicolumn{2}{|c|}{ ORA and NTA } & \multicolumn{2}{|c|}{ Competitive gene set test } \\
\hline & & & Terms $(n)$ & FDR (median) & Genes $(n)$ & FDR (exact) \\
\hline & & Cell migration and chemotaxis & 77 & $1.1 \mathrm{e}-04$ & 290 & $9.9 e-05$ \\
\hline & & Signal transduction & 73 & $2.8 \mathrm{e}-06$ & 723 & $5.5 e-05$ \\
\hline & & Tissue development and regeneration & 67 & $2.1 \mathrm{e}-04$ & 925 & $1.7 e-06$ \\
\hline & & Ubiquitination and apoptosis & 63 & $6.5 e-04$ & 472 & $3.3 e-04$ \\
\hline & & Biosynthetic process & 54 & $1.0 \mathrm{e}-03$ & 558 & $1.1 e-03$ \\
\hline & & Cell-cell adhesion & 49 & $3.1 e-05$ & 499 & $1.9 e-05$ \\
\hline & & Synaptic function and activity & 38 & $2.1 e-04$ & 171 & $3.7 e-05$ \\
\hline & & Phagocytosis and endocytosis & 36 & $1.7 \mathrm{e}-05$ & 242 & $1.7 e-03$ \\
\hline & & Microtubuli and cytoskeleton & 32 & $6.1 e-04$ & 508 & 2.7e-02 \\
\hline & & Cell membrane activity & 31 & $3.1 \mathrm{e}-06$ & 670 & $1.1 \mathrm{e}-04$ \\
\hline & & Regulation of (cell) proliferation & 29 & $2.6 e-05$ & 429 & $1.8 \mathrm{e}-04$ \\
\hline & & Regulation of (cell) differentiation & 25 & $1.5 e-03$ & 506 & $1.3 e-05$ \\
\hline & & Transcriptional activity & 18 & $3.0 \mathrm{e}-03$ & 356 & $1.6 e-04$ \\
\hline & & Catabolic process & 17 & $5.5 e-03$ & 233 & $1.3 e-03$ \\
\hline & & Cell and tissue morphology & 15 & 4.6e-03 & 275 & $2.3 e-05$ \\
\hline & & Regulation of (cell) activation & 12 & $5.0 e-10$ & 252 & $2.2 \mathrm{e}-03$ \\
\hline & & I-kappaB kinase/NF-kappaB signaling & 11 & $5.9 e-04$ & 70 & $2.3 e-03$ \\
\hline & & Secretion & 11 & $2.3 e-03$ & 89 & $1.8 \mathrm{e}-04$ \\
\hline & & Neuronal cell activity & 10 & $2.4 \mathrm{e}-07$ & 366 & 2.7e-05 \\
\hline & & Sensory perception process & 10 & $1.8 \mathrm{e}-03$ & 59 & 4.7e-05 \\
\hline \multirow[t]{2}{*}{ MSC[SCI] vs MSC[Naive] } & Up & Tissue development and regeneration & 52 & 8.7e-04 & 43 & $6.3 e-04$ \\
\hline & & Cell morphology & 27 & $9.6 e-04$ & 36 & $9.6 e-04$ \\
\hline
\end{tabular}

ORA over-representation enrichment analysis, NTA network topology-based analysis. Terms: unique terms identified using Gene Ontology (GO)-biological process, GO-molecular function, GO-cellular component, Kyoto Encyclopedia of Genes and Genomes (KEGG), network retrieval and prioritization (NRP), network expansion (NE). Genes: unique genes constituting the (unique) GO and KEGG terms for a specific category. Categories with a total of less than ten unique GO/KEGG terms are omitted from the table for enhanced interpretation

cytokine production/response, immune system cells, and phagocytosis/endocytosis, suggesting that transplanted MSCs adopt immune cell-like characteristics.

\section{Transplanted MSCs express CD29, Sca1, and CD45}

Phenotypic characterization of positive and negative surface markers of MSCs was performed at 7 days following transplantation (Fig. 2a). A majority of non-transplanted MSCs expressed CD29 and Sca1 (Additional file 4: Figure S4C3, C4, C6). Transplanted MSCs maintained expression of CD29 and Sca1 in both injured (88.2\%, CI 87.9-88.5\%) (Fig. 2b, c) and uninjured (90.9\%, CI 87.993.9\%) spinal cord $(p<0.05)$ (Fig. 2b, d). Transplanted MSCs had a similar but slightly lower gene expression of CD29 (SCI - 0.58, naive - 0.85) as compared to non-transplanted MSCs $(p<0.01)$ (Fig. 2e). However, transplanted MSCs upregulated their gene expression of Sca1 (SCI 2.87, naive 2.57) as compared to non-transplanted MSCs $(p<0.001)$ (Fig. 2e). MSCs did not express CD45 in culture conditions (Additional file 4: Figure S4C5, C7, C8). Following transplantation, a majority of Sca1+ MSCs expressed CD45 in the injured (59.5\%, CI 58.6-60.3\%) and uninjured (58.2\%, CI 56.2$60.3)$ spinal cord $(p<0.05)$ (Fig. $2 \mathrm{~b}-\mathrm{d})$. This was also true for CD29+ MSCs in both injured (66.0\%, CI 64.6-67.4\%) and uninjured (63.1\%, CI 58.8-67.4\%) spinal cord $(p<0.05)$ (Fig. 2b-d). Transplanted MSCs significantly upregulated their gene expression of CD45 (SCI 12.81, naive 12.26) as compared to non-transplanted MSCs $(p<0.001)$ (Fig. 2e). Cells expressing Cx3cr1 aggregated around the grafted MSCs but did not infiltrate the graft at 7 days after transplantation (Additional file 5: Figure S5). Taken together, MSCs transplanted into the uninjured or injured spinal cord have a surface and gene expression of Sca1 and CD29 comparable to non-transplanted MSCs but significantly upregulate surface and gene expression of CD45 as compared to non-transplanted immunodepleted MSCs.

\section{Transplanted MSCs express MHC-I and MHC-II}

MSCs in culture express MHC-I but not MHC-II on the cell surface. Ninety percent of MSCs exposed to IFN- $\gamma$ for $48 \mathrm{~h}$ in culture express MHC-II on the cell surface 


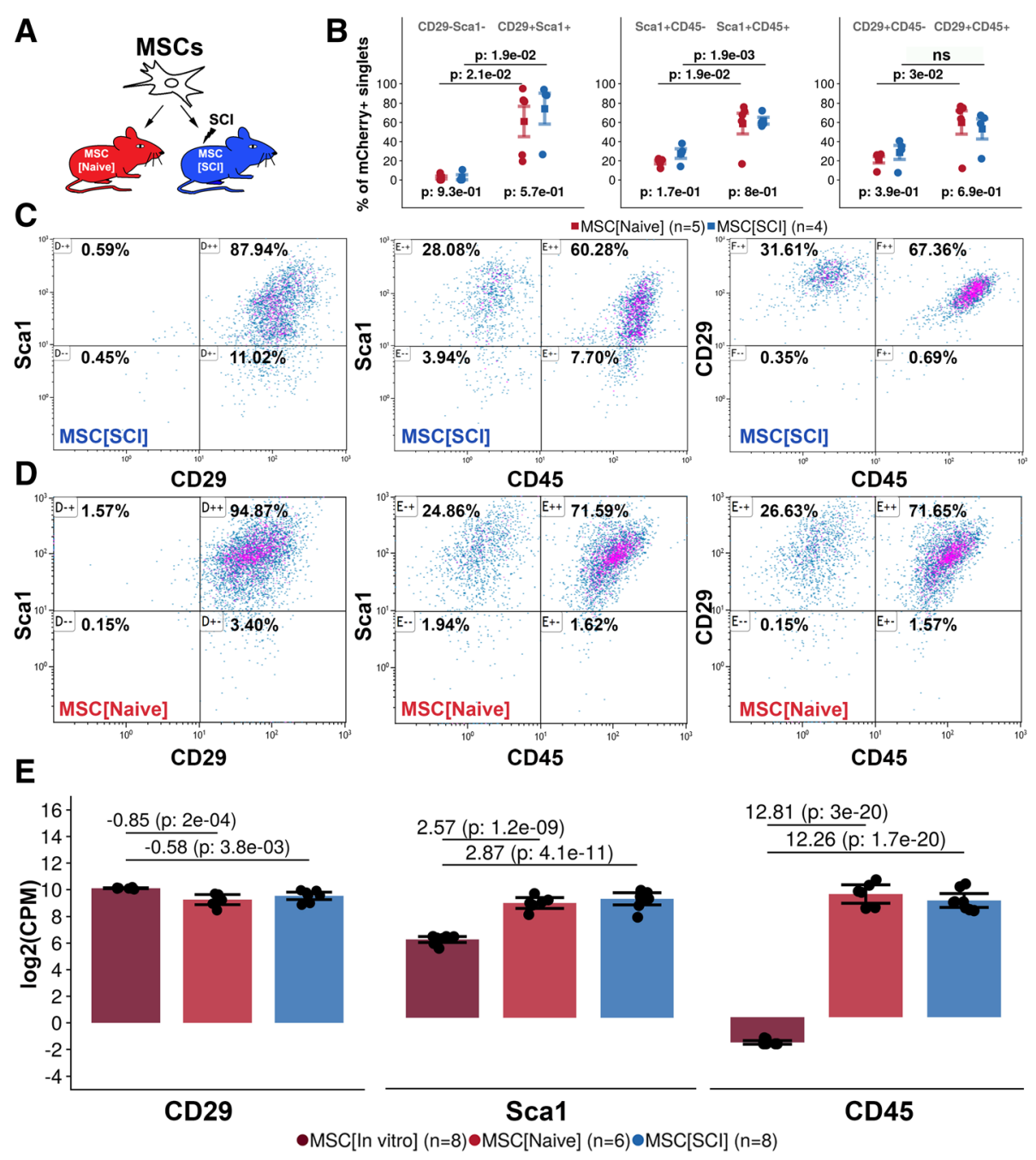

Fig. 2 Expression of CD29, Sca1, and CD45 in transplanted MSCs. a Experimental design. Transplanted MSCs were evaluated 7 days following transplantation. b Gate statistics for each experimental group and relevant gates. Mean is surrounded by a $95 \%$ confidence interval. Each dot represents one biological replicate. Values above plot represent $p$ values following independent two-group comparison within study groups between gates. Values below plot represent $p$ values following independent two-group comparison within gate between study groups. c Representative bivariate plots of MSC[SCI]. d Representative bivariate plots of MSC[Naive]. e Log2 (counts per million) for CD29, Sca1, and CD45 genes following global transcriptional analysis of MSCs. Dots represent biological replicates. Mean is surrounded by $95 \%$ confidence interval. Log2(fold change) followed by the FDR for contrasts: MSC[SCI] vs MSC[In vitro] and MSC[Naive] vs MSC[In vitro] is presented in the plot

[27]. We hypothesized that the same type of upregulation of MHC-II on the cell surface could occur in MSCs transplanted into SCI (Fig. 3a). We confirmed that MSCs in culture express MHC-I (37.3\%, CI 37.0-37.5\%) but not MHC-II (1.7\%, CI 1.6-1.8\%) (Fig. 3b, c, e). Expression of MHC-I on the cell surface of transplanted MSCs was not significantly altered following exposure to the injured $(16.9 \%$, CI $3.1-30.6 \%)$ or uninjured $(15.6 \%$, CI 4.9-26.2\%) spinal cord compared to MSCs in culture (Fig. 3b, c). Transplanted MSCs upregulated gene expression of MHC-I (SCI 2.34, naive 2.51) as compared to non-transplanted MSCs $(p<0.001)$ (Fig. 3d). In comparison, MSCs transplanted to the injured (7.6\%, CI 6.7$8.4 \%)$ or uninjured $(6.0 \%$, CI $5.5-6.4 \%)$ spinal cord had a higher surface expression of MHC-II as compared to MSCs in culture $(p<0.01)$ (Fig. 3b, e). Gene expression analysis confirmed that MHC-II was significantly upregulated in transplanted MSCs (SCI 9.25, naive 9.14) as compared to non-transplanted MSCs $(p<0.001)$ (Fig. 3f). Histological evaluation of transplanted MSCs confirmed co-expression of mCherry and MHC-II in both injured and uninjured spinal cord (Fig. 3g). The percentage mCherry+MHC-II+MSCs was not significantly different between MSCs in the injured (7.2\%, CI 4.5-9.9\%) and uninjured spinal cord (11.0\%, CI 3.3-18.6\%) but comparable to findings in Fig. 3e (Fig. 3h). Taken together, MSCs transplanted into the injured or uninjured spinal cord maintain their surface and gene expression of 


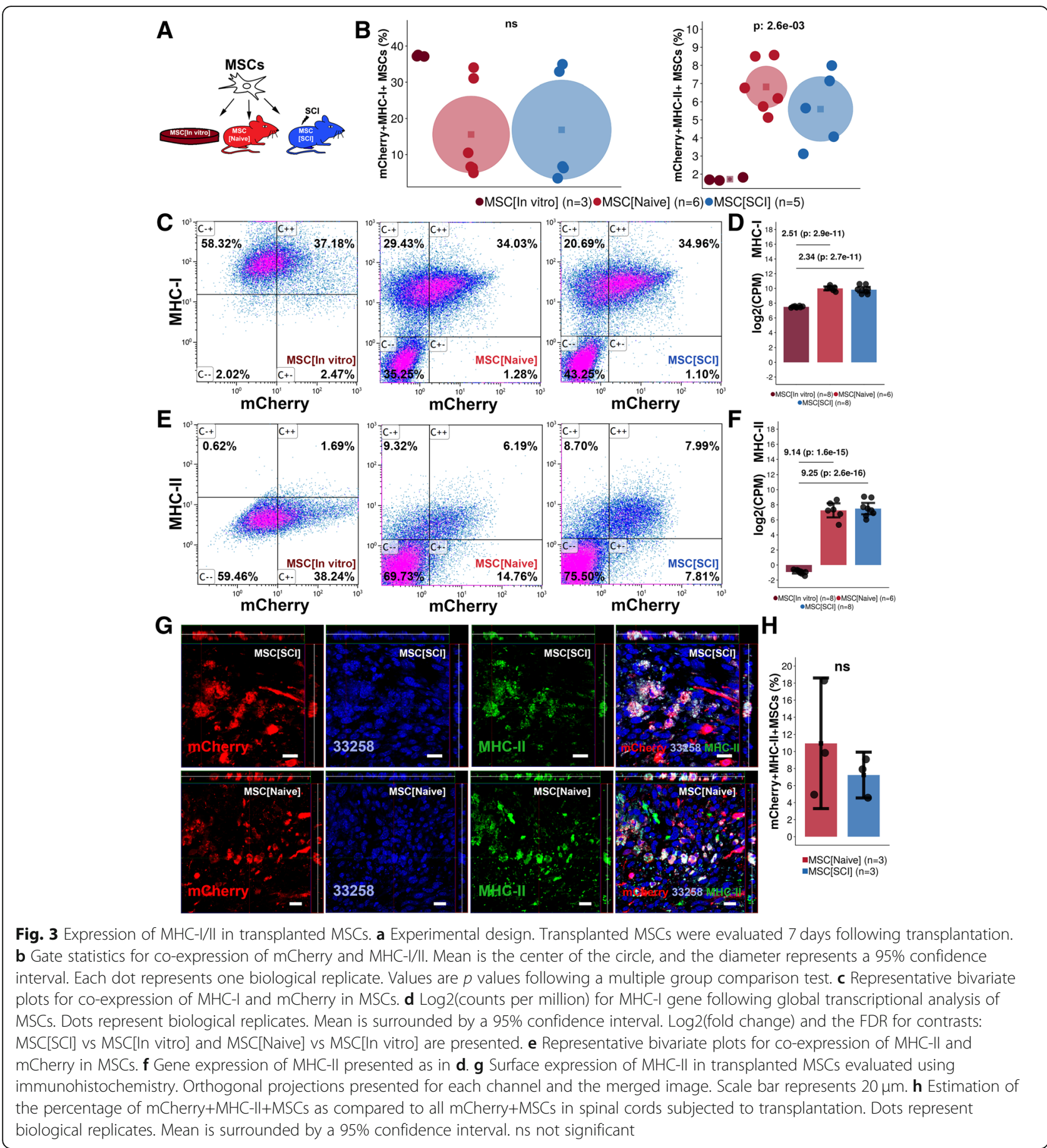

MHC-I but upregulate surface and gene expression of MHC-II in comparison to non-transplanted MSCs.

Transplanted MSCs survive and proliferate but do not undergo apoptosis or neural differentiation

Survival, proliferation, apoptosis, and differentiation of transplanted MSCs were assessed at 7 and 14 days post transplantation (Figs. 4a and 5a). Transplanted MSCs could be detected for 7 but not 14 days in the uninjured
(261, CI 167-355) and injured (335, CI 195-476) spinal cord, respectively (Fig. 4b). The spinal cord environment did not affect MSC survival. MSCs proliferated to a low extent in both uninjured (6.9\%, CI 1.1-12.8\%) (Fig. 4c, f) and injured (3.7\%, CI 1.5-5.9\%) (Fig. 4c, g) spinal cord. Although the proliferation tended to be higher for MSCs exposed to the uninjured spinal cord, no significant difference could be detected between the experimental groups. The proliferation rate of transplanted MSCs was 


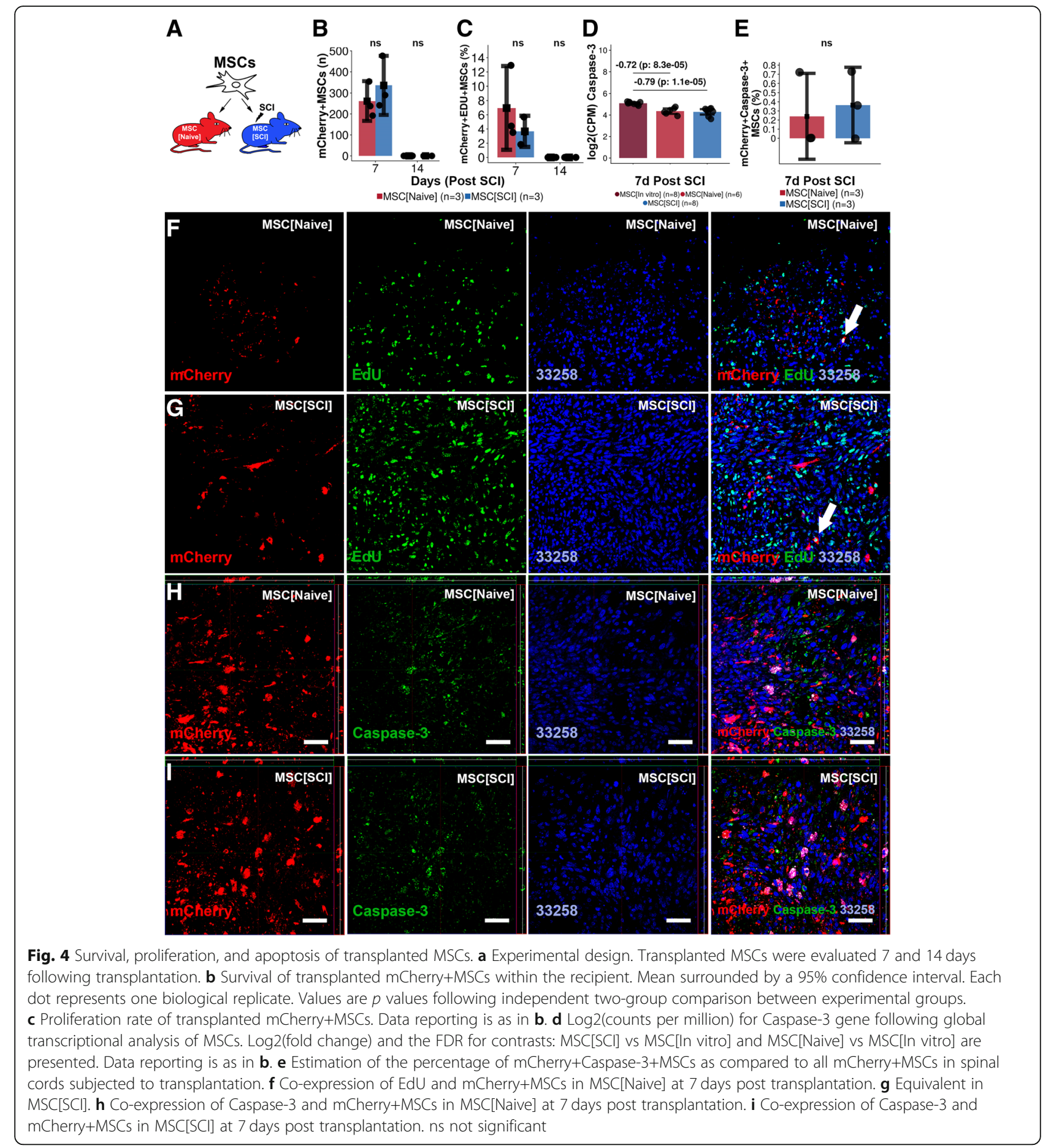

about one ninth of the proliferation rate in culture (Additional file 4: Figure S4B1-B3). MSCs downregulated gene expression of Caspase-3 following transplantation into both injured $(-0.79)$ and uninjured spinal cord $(-0.72)(p<0.001)$ (Fig. 4d). Histological examination revealed no relevant co-expression between mCherry+MSCs and Caspase- 3 in the injured $(0.4 \%, \mathrm{CI}-0.05-0.8 \%)$ and uninjured spinal cord
(0.2\%, CI $-0.2-0.7 \%)$ (Fig. 4e, h, i) using positive control as the reference (Additional file 6: Figure S6). No significant difference in the number of mCherry +Caspase-3+MSCs could be detected between MSCs in the injured and uninjured spinal cord (Fig. 4e). Gene expression analysis revealed an upregulation of $\mathrm{TuJ} 1$ in MSC[SCI] as compared to MSC[In vitro] (2.17, $p<0.001$ ) while MSC[Naive] did not have an 

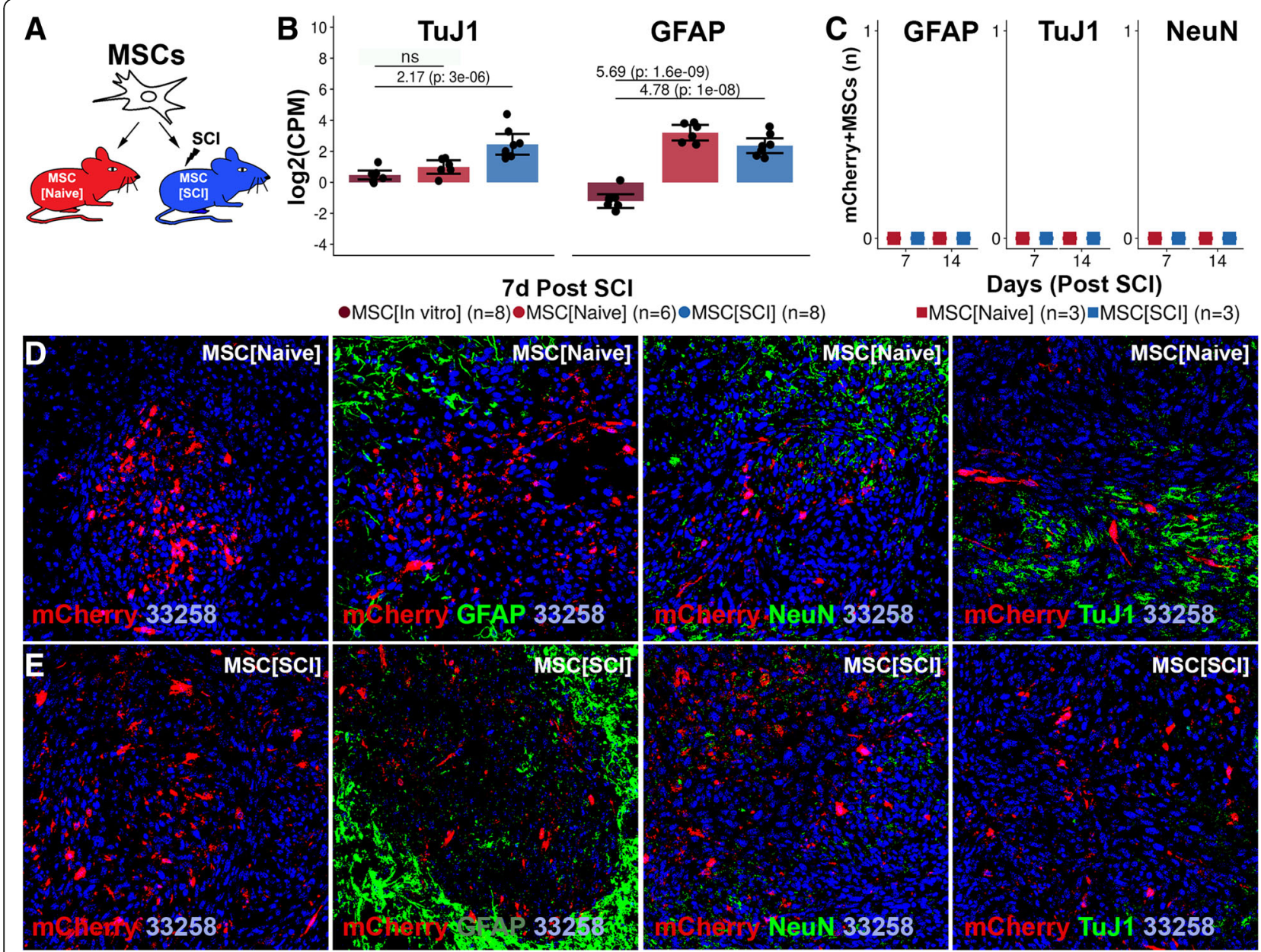

Fig. 5 Differentiation of transplanted MSCs. a Experimental design. Transplanted MSCs were evaluated 7 and 14 days following transplantation. b Log2(counts per million) for TuJ1 and GFAP gene following the global transcriptional analysis of MSCs. NeuN gene was filtered out due to low expression. Log2(fold change) and the FDR for contrasts: MSC[SCI] vs MSC[In vitro] and MSC[Naive] vs MSC[In vitro] are presented. Mean surrounded a 95\% confidence interval. Each dot represents one biological replicate. c Co-expression of mCherry+MSCs and GFAP, NeuN, and TuJ1, respectively. d Co-expression of GFAP, NeuN, TuJ1, and mCherry+MSCs in MSC[Naive] at 7 days post transplantation. e Equivalent to $\mathbf{d}$ for MSC[SCI]

upregulation (0.66, $p=0.1$ ) (Fig. 5b). However, MSCs upregulated gene expression of GFAP in both injured (4.78, $p<0.001)$ and uninjured spinal cord (5.69, $p<0.001$ ). Histological examination of co-expression between mCherry and GFAP, NeuN, or TuJ1 revealed no double positive cells in neither uninjured (Fig. $5 \mathrm{c}$, d) nor injured (Fig. 5c, e) spinal cord, suggesting a lack of detectable neuronal or astrocytic differentiation. Taken together, MSCs transplanted into the uninjured or injured spinal cord survive for 1 week and proliferate to a low extent but do not undergo neural or astrocytic differentiation.

\section{Discussion}

In this study, we investigated the cellular response of MSCs following transplantation into spinal cord injury (SCI). We found that MSCs transplanted into SCI upregulate expression of CD45 and MHC-II and expression of genes related to cytokine production, phagocytosis/endocytosis, and immune cells/response and thereby adopt immune cell-like characteristics within the recipient.

Transplanted MSCs were detected in both injured and uninjured spinal cord at 7 , but not 14 , days following transplantation. The short-term survival is in line with the findings of Jung et al. [28] and others [13, 28-31]. However, it was not within the scope of this study to determine whether the fluorescence faded or if the MSCs perished. Considering that a commonly available expression construct was implemented, the fluorescence did not fade in culture conditions, and other authors have reported short-term survival, we confidently hypothesize that the MSCs perish between 7 and 14 days following transplantation. Hence, the lower limit of survival seems to be 7 days, but the upper limit remains uncertain. Even though the Caspase-3 expression in transplanted MSCs was negligible, the global transcriptional analysis 
revealed that transplanted MSCs significantly upregulated genes and GO/KEGG terms related to ubiquitination and apoptosis. In the few cells which were observed and reported as Caspase- 3 positive, the staining was sometimes difficult to distinguish from phagosomes. However, considering that the global transcriptional analysis revealed an upregulation of genes related to phagocytosis, it is likely that the MSCs did indeed have phagosomes, which confirms their adoption of immune cell-like characteristics. Hence, the lack of detectable MSCs at 14 days post transplantation is likely cell death resulting from the challenging environment. Additionally, the downregulation of genes related to DNA repair in transplanted MSCs, in combination with the downregulation of genes related to cell-cycle activity, supports the theory that MSCs are struggling to survive within the recipient and simply perish between 7 and 14 days post transplantation. Given that the MSCs manage to secrete enough tissue and immunomodulatory factors [14, $15,21,32-40]$ during this time to enhance recovery, the short-term survival is not of great concern. However, it is interesting to speculate whether long-term survival could enhance recovery further. Transplanted MSCs did proliferate in both injured and uninjured spinal cord, but at about one ninth of the rate in culture. This is in line with the findings in the global transcriptional analysis which indicated that genes related to cell-cycle progression/regulation were downregulated and detected using EdU in a small number of cells. This in combination with the downregulation of genes related to DNA processes and repair indicates that the MSCs are active within the recipient but slowing down. The lower rate of proliferation in vivo as compared to in culture is most likely also a consequence of the hazardous SCI environment limiting the nutrition and oxygen supply and resulting in a less beneficial growth environment. Transplanted MSCs did not differentiate in the injured or uninjured spinal cord. Since MSCs are from mesodermal and not ectodermal origin, this result was expected. However, Aras et al. [4] and others found that transplanted MSCs could differentiate into astrocytes [12, 41, 42], oligodendrocytes $[4,43]$, and neurons $[4,6,9]$. Interestingly however, the global transcriptional analysis revealed that MSCs in SCI upregulated gene expression of TuJ1 and GFAP, which is more in line with previous reports. The lack of differentiation detected in this study might be due to the time point of evaluation, the tissue from which the MSCs were established, the severity of the injury, the type of injury, or a similar factor. However, most authors in the field are convinced that MSCs mediate their beneficial modulation of the microenvironment mainly by paracrine effects $[2,10,13,28]$. Assuming that this is true, the lack of ectodermal differentiation is of minor importance. Taken together, MSCs survive for a brief period of time during which the MSCs do not undergo apoptosis but a fraction is active and proliferate. Moreover, MSCs do not undergo neural differentiation following transplantation, which supports the theory that MSCs act through tissue and immune system modulation early on following transplantation rather than trans-differentiation and integration.

Transplanted MSCs maintained their expression of CD29 and Sca1 but also expressed CD45 on the cell surface, which was in line with gene expression analysis. Hence, MSCs preserve stem cell characteristics but adopt an immune cell-like phenotype following transplantation. CD45 expression could indicate that the MSCs respond to the in vivo environment and therefore alter their phenotypic profile. The fact that the MSCs did express CD29 and Sca1 to a high extent indicates that the CD45 expression is not a result of the MSCs being phagocytized by macrophages. Transplanted MSCs maintained their expression of MHC-I but upregulated expression of MHC-II detected using flow cytometry, immunohistochemistry, and gene expression analysis. MSCs in culture conditions express MHC-II within the cell but not on the cell surface [27, 44]. Le Blanc et al. [27] demonstrated that MSCs in culture, exposed to IFN $\gamma$ for $48 \mathrm{~h}$, express MHC-II on the cell surface. IFN $\gamma$ is critical for the innate and adaptive immune response and is present in a SCI environment $[45,46]$. Thus, the ability of IFNY to induce MHC-II expression seems to be true not only in vitro but also in vivo. Moreover, MHC-II is a macrophage marker which further supports that MSCs adopt an immune cell-like phenotype. The histological evaluation clearly demonstrated the MHC-II expression. Careful inspection of the MSCs reveal structures which might resemble phagosomes, which is in line with and supports the MHC-II expression considering that MSCs transform into macrophage-like cells and should gain the ability to phagocytize, which was also predicted by the global transcriptional analysis. The fact that MSCs maintain their CD29 and Sca1 expression significantly lowers the probability of the MSCs being engulfed by macrophages and rather supports the fact that the cells are still MSCs, but with an immune cell-like phenotype and function and they themselves phagocytize. This is further supported by the fact that the immune cells clearly surrounded the graft but did not enter into it as revealed by Additional file 5: Figure S5. Furthermore, global transcriptional analysis revealed that MSCs upregulate genes related to immune system response, phagocytosis/endocytosis, and cytokine production and release, which further supports the fact that MSCs seem to take an immune cell-like phenotype following transplantation. Genes related to both the innate and the adaptive immune system but also to leukocytes (CD45+ cells) were 
identified. The upregulation of genes related to production and release of pro-inflammatory cytokines further suggests that MSCs take a macrophage-like profile with secretory capabilities. Furthermore, this sheds new light on the assumed immune privilege of MSCs and might have to be taken into account in MSC-based therapies, not limited to SCI. Taken together, transplanted MSCs upregulate expression of CD45 and MHC-II and thereby adopt an immune cell-like phenotype but also upregulate genes related to cytokine production, immune cells/response, and phagocytosis/endocytosis indicating that the MSCs also undertake immune cell-like functions and that this is induced by the SCI environment.

Cytokine expression is usually upregulated in both the spinal cord and the cerebrospinal fluid following SCI as a result of activation of macrophages. We found that genes relating to cytokine stimulus response and production were upregulated in MSCs transplanted into $\mathrm{SCI}$ and that pathways related to TNF $\alpha$ were affected specifically. Both IL-1 $\beta$ [47] and TNF $\alpha$ [48] activate NF- $\kappa \beta$-dependent transcription [49]. The increased response to cytokines coincided with significant upregulation of genes related to signaling pathways, especially NF- $\kappa \beta$ signaling pathways. MSCs upregulated GO terms related to increased response to IFN $\gamma$ which might be related to the elevated MHC-II expression on MSCs, as indicated by Le Blanc et al. [27].

Extracellular vesicles are secreted from endosomal compartments of MSCs (MSC-EVs) [50, 51] and have been characterized as mediators of the immune-modulatory effects of MSCs [15, 32-40]. Assuming that MSCs mediate the majority of their therapeutic effect through paracrine effects, the content of the MSC-EVs is highly interesting to investigate. But, it is reasonable to hypothesize that at least some of the transcriptional changes induced in MSCs by the in vivo environment are transmitted to the surrounding tissue using MSC-EVs. Identifying, isolating, and sequencing MSC-EVs released by transplanted MSCs would potentially reveal the full mechanism of action of MSCs. Given that the cargo-or at least essential parts of the cargo-could be constructed synthetically and administrated directly to a patient, the need for MSC transplantation could perhaps be abolished. This study however aimed at investigating the cellular response of transplanted MSCs and did not aim to identify any potential therapeutic agents secreted by the MSCs.

The low number of genes significantly differentially expressed between MSC[Naive] and MSC[SCI] indicated a similarity between these two experimental groups. The comparison between these two and MSC[In vitro] indicated up- and downregulation of the same type of functional categories further emphasizing their similarity.
Evaluation of phenotypes, proliferation, survival, and differentiation also indicated a striking similarity between MSC[SCI] and MSC[Naive]. The similarity between $\mathrm{MSC}[\mathrm{SCI}]$ and MSC[Naive] is probably a consequence of the non-significant injury caused by the glass capillary needle during transplantation. This is not only a limitation in this study, but also a limitation in stem cell transplantation as a therapeutic approach for SCI. Furthermore, the results presented in this study might vary with the time point of evaluation, the severity of the injury, the injury type, the species, and/or the strain. One additional crucial limitation to keep in mind in all transplantation studies is transplantation accuracy. Furthermore, the results in this study were not adjusted for differences in force, displacement, and/or velocity of the SCI.

\section{Conclusion}

In this study, we found that MSCs transplanted into SCI upregulate expression of CD45 and MHC-II and expression of genes related to cytokine production, phagocytosis/endocytosis, and immune cells/response and thereby adopt immune cell-like characteristics within the recipient. Understanding the cellular response of MSCs allows for modification, enhancement, and/or replacement of the effect mediated by MSCs. This could lead the way toward not only a more efficient therapy, but perhaps also a cell-free therapy that could be beneficial in terms of recovery, time, effort, and cost.

\section{Additional files}

\begin{abstract}
Additional file 1: Figure S1. MSCs pre- and post transplantation. Figure S1A, B mCherry+MSCs prior to transplantation ( $48 \mathrm{~h}$ following transfection). Figure S1C mCherry+MSCs transplanted, sorted (FACS) following 7 days in the recipient, plated and kept in culture for $72 \mathrm{~h}$. Fluorescence of mCherry in MSCs evaluated using excitation at wavelength $633 \mathrm{~nm}$. Figure S1D co-expression of mCherry and Sca1 in MSCs isolated from spinal cord 7 days following transplantation. (TIF 27481 kb)
\end{abstract}

Additional file 2: Figure S2. Physical parameters and gating strategy of transplanted MSCs. Figure S2A-C non-transplanted non-transfected MSCs. Figure S2D-F non-transplanted transfected MSCs. Figure S2G-I uninjured spinal cord. Figure S2J-L uninjured spinal cord with transplanted transfected MSCs. Figure S2M-O injured spinal cord. Figure S2P-R injured spinal cord with transplanted transfected MSCs. (TIF $46283 \mathrm{~kb}$ )

Additional file 3: Figure S3. Global transcriptional changes in MSCS extended analysis. Figure S3A two first components/variables following dimensionality reduction using principal component analysis (PCA) and t-distributed stochastic neighbor embedding ( $\mathrm{tSNE}$, perplexity $=3$, theta $=0.5$ ). Each dot represents one biological replicate. Ellipse represents 95\% confidence interval. Figure S3B bootstrapped (1000 runs) first three components of PCA clustered using affinity propagation (left) and k-means clustering (right, 3 clusters, 20 starts). Figure S3C two first components following PCA and tSNE clustered using K-means clustering (KM, 3 clusters, 20 starts), affinity propagation (AP), expectation maximum (EM), and K-nearest neighbor (KNN, 60:40 split). Figure S3D top positive and negative loadings for the first and second principal component following PCA. (TIF 23979 kb) 
Additional file 4: Figure S4. Proliferation and expression of CD29, Sca1 and CD45 in non-transplanted MSCs. Figure S4A experimental design. Figure S4B1-B3 expression of EdU in mCherry+MSCs. Figure S4C1-C5 expression of CD29, Sca1, and CD45 in mCherry+MSCs. Figure S4C6-C8 co-expression of CD29, Sca1, and CD45 in mCherry+MSCs. (TIF $16399 \mathrm{~kb}$ )

Additional file 5: Figure S5. Interaction between transplanted MSCs and immune cells. MSCs in relation to immune cells at 7 days following transplantation into injured spinal cord. (TIF $6636 \mathrm{~kb}$ )

Additional file 6: Figure S6. Caspase-3 positive control. Mouse liver tissue stained with Caspase-3 acting as positive control to Caspase-3 staining of mCherry+MSCs. Scale bar represents $20 \mu \mathrm{m}$. (TIF $894 \mathrm{~kb}$ )

\section{Abbreviations}

SCl: Spinal cord injury; MSC: Mesenchymal stem cell;

MSC[Naive]: Mesenchymal stem cells transplanted into an uninjured spinal cord; MSC[SCI]: Mesenchymal stem cells transplanted into an injured spinal cord; MSC[In vitro]: Non-transplanted mesenchymal stem cells; ORA: Overrepresentation enrichment analysis; NTA: Network topology-based analysis; GO: Gene Ontology; KEGG: Kyoto Encyclopedia of Genes and Genomes; BP: Biological process (GO); CC: Cellular component (GO); MF: Molecular function (GO); FDR: False discovery rate; LogFC: Log2(fold change); Log2(CPM): Log2(counts per million); EV: Extracellular vesicle; PCA: Principal component analysis; tSNE: $t$-distributed stochastic neighbor embedding

\section{Acknowledgements}

We appreciate contributions from all group members in the BrundinSvensson (Karolinska Institutet, KI) and Tomas Olsson research group (KI); Annika van Vollenhoven (KI) for excellent support with FACS; the National Genomics Infrastructure funded by Science for Life Laboratory, the Knut and Alice Wallenberg Foundation, and the Swedish Research Council; and SNIC/ Uppsala multidisciplinary center for advanced computational science for assistance with massive parallel sequencing and access to the UPPMAX computational infrastructure.

\section{Funding}

The Swedish Medical Research Council, The Swedish Society of Medicine, Karolinska Institutet, Swedish Brain Foundation, Stockholm City Council.

\section{Availability of data and materials}

The datasets used and/or analyzed during the current study are available from the corresponding author on reasonable request.

\section{Authors' contributions}

$R H, R C, V Z, A F, S S, L B$, and $M S$ contributed to the experimental design. $R H$, $R C, V Z, A F, S S, L B$, and MS contributed to the manuscript writing and editing. $\mathrm{RH}$ contributed to the animal care, spinal cord injury and cell transplantation, sacrifice, and establishment of cell cultures and cell culturing. RH, RC, and SS contributed to the fluorescence-activated cell sorting. $\mathrm{RH}$ and SS contributed to the RNA isolation. RH and VZ contributed to the RNA sequencing. RH and $\mathrm{RC}$ contributed to the flow cytometry and proliferation assay. RH and SS contributed to the immunohistochemistry and immunocytochemistry. RH contributed to the differentiation assay, confocal microscopy, automatic cell quantification, and data and statistical analysis. All authors read and approved the final manuscript.

\section{Ethics approval and consent to participate}

Ethical permits were obtained from the local ethics committee (Stockholm, Sweden): N196/15, N12317-2017, and N38/16.

\section{Consent for publication}

Not applicable.

\section{Competing interests}

The authors declare that they have no competing interests.

\section{Publisher's Note}

Springer Nature remains neutral with regard to jurisdictional claims in published maps and institutional affiliations.

\section{Author details}

'Department of Neurology, Karolinska University Hospital, 17176 Stockholm, Sweden. ${ }^{2}$ Center for Molecular Medicine, Karolinska Institutet, 17176 Stockholm, Sweden. ${ }^{3}$ Department of Clinical Neuroscience, Karolinska Institutet, 17176 Stockholm, Sweden. ${ }^{4}$ Department of Oncology and Pathology, Karolinska Institutet, 17176 Stockholm, Sweden. ${ }^{5}$ BioClinicum, Karolinska University Hospital, Solnavägen 30, Solna, 17164 Stockholm, Sweden. ${ }^{6}$ Department of Neurosurgery, Karolinska University Hospital, 17176 Stockholm, Sweden.

Received: 21 November 2018 Revised: 27 February 2019 Accepted: 20 March 2019 Published online: 03 April 2019

\section{References}

1. Okuda A, Horii-Hayashi N, Sasagawa T, Shimizu T, Shigematsu H, Iwata E, Morimoto Y, Masuda K, Koizumi M, Akahane M, et al. Bone marrow stromal cell sheets may promote axonal regeneration and functional recovery with suppression of glial scar formation after spinal cord transection injury in rats. J Neurosurg Spine. 2017;26(3):388-95.

2. Zhou HL, Zhang XJ, Zhang MY, Yan ZJ, Xu ZM, Xu RX. Transplantation of human amniotic mesenchymal stem cells promotes functional recovery in a rat model of traumatic spinal cord injury. Neurochem Res. 2016:41(10):2708-18.

3. Morita T, Sasaki M, Kataoka-Sasaki Y, Nakazaki M, Nagahama H, Oka S, Oshigiri T, Takebayashi T, Yamashita T, Kocsis JD, et al. Intravenous infusion of mesenchymal stem cells promotes functional recovery in a model of chronic spinal cord injury. Neuroscience. 2016;335:221-31.

4. Aras Y, Sabanci PA, Kabatas S, Duruksu G, Subasi C, Erguven M, Karaoz E. The effects of adipose tissue-derived mesenchymal stem cell transplantation during the acute and subacute phases following spinal cord injury. Turk Neurosurg. 2016;26(1):127-39.

5. Torres-Espín A, Redondo-Castro E, Hernandez J, Navarro X. mmunosuppression of allogenic mesenchymal stem cells transplantation after spinal cord injury improves graft survival and beneficial outcomes. J Neurotrauma. 2015:32(6):367-80.

6. Chen YB, Jia QZ, Li DJ, Sun JH, Xi S, Liu LP, Gao DX, Jiang DW. Spinal cord injury in rats treated using bone marrow mesenchymal stem-cell transplantation. Int J Clin Exp Med. 2015;8(6):9348-54.

7. Chung HJ, Chung WH, Lee JH, Chung DJ, Yang WJ, Lee AJ, Choi CB, Chang $\mathrm{HS}$, Kim DH, Suh HJ, et al. Expression of neurotrophic factors in injured spinal cord after transplantation of human-umbilical cord blood stem cells in rats. J Vet Sci. 2016;17(1):97-102.

8. Matsushita T, Lankford KL, Arroyo EJ, Sasaki M, Neyazi M, Radtke C, Kocsis JD. Diffuse and persistent blood-spinal cord barrier disruption after contusive spinal cord injury rapidly recovers following intravenous infusion of bone marrow mesenchymal stem cells. Exp Neurol. 2015;267:152-64.

9. Cui B, Li E, Yang B, Wang B. Human umbilical cord blood-derived mesenchymal stem cell transplantation for the treatment of spinal cord injury. Exp Ther Med. 2014;7(5):1233-6.

10. de Almeida FM, Marques SA, BoS R, Massoto TB, Martinez AM. Chronic spinal cord lesions respond positively to tranplants of mesenchymal stem cells. Restor Neurol Neurosci. 2015;33(1):43-55.

11. Oda Y, Tani K, Asari Y, Quintanilha LF, Haraguchi T, Momota Y, Katayama M, Itamoto K, Nakazawa H, Taura Y. Canine bone marrow stromal cells promote functional recovery in mice with spinal cord injury. J Vet Med Sci. 2014;76(6):905-8.

12. Lee KH, Suh-Kim H, Choi JS, Jeun SS, Kim EJ, Kim SS, Yoon DH, Lee BH. Human mesenchymal stem cell transplantation promotes functional recovery following acute spinal cord injury in rats. Acta Neurobiol Exp (Wars). 2007;67(1):13-22

13. Zhou Z, Chen Y, Zhang H, Min S, Yu B, He B, Jin A. Comparison of mesenchymal stromal cells from human bone marrow and adipose tissue for the treatment of spinal cord injury. Cytotherapy. 2013;15(4):434-48.

14. Li Y, Gao X, Wang J. Human adipose-derived mesenchymal stem cellconditioned media suppresses inflammatory bone loss in a lipopolysaccharide-induced murine model. Exp Ther Med. 2018;15(2): 1839-46.

15. Ahn SY, Park WS, Kim YE, Sung DK, Sung SI, Ahn JY, Chang YS. Vascular endothelial growth factor mediates the therapeutic efficacy of mesenchymal stem cell-derived extracellular vesicles against neonatal hyperoxic lung injury. Exp Mol Med. 2018;50(4):26. 
16. Collino F, Deregibus MC, Bruno S, Sterpone L, Aghemo G, Viltono L, Tetta C, Camussi G. Microvesicles derived from adult human bone marrow and tissue specific mesenchymal stem cells shuttle selected pattern of miRNAs. PLoS One. 2010;5(7):e11803.

17. Eirin A, Zhu XY, Puranik AS, Woollard JR, Tang H, Dasari S, Lerman A, van Wijnen AJ, Lerman LO. Integrated transcriptomic and proteomic analysis of the molecular cargo of extracellular vesicles derived from porcine adipose tissue-derived mesenchymal stem cells. PLoS One. 2017;12(3):e0174303.

18. Nargesi AA, Lerman LO, Eirin A. Mesenchymal stem cell-derived extracellular vesicles for renal repair. Curr Gene Ther. 2017;17(1):29-42.

19. Yu S, Zhao Y, Ma Y, Ge L. Profiling the secretome of human stem cells from dental apical papilla. Stem Cells Dev. 2016;25(6):499-508.

20. Johnson TV, DeKorver NW, Levasseur VA, Osborne A, Tassoni A, Lorber B, Heller JP, Villasmil R, Bull ND, Martin KR, et al. Identification of retinal ganglion cell neuroprotection conferred by platelet-derived growth factor through analysis of the mesenchymal stem cell secretome. Brain. 2014; 137(Pt 2):503-19.

21. Szekiova E, Slovinska L, Blasko J, Plsikova J, Cizkova D. The neuroprotective effect of rat adipose tissue-derived mesenchymal stem cell-conditioned medium on cortical neurons using an in vitro model of SCl inflammation. Neurol Res. 2018;40(4):258-67.

22. Marote A, Teixeira FG, Mendes-Pinheiro B, Salgado AJ. MSCs-derived exosomes: cell-secreted nanovesicles with regenerative potential. Front Pharmacol. 2016;7:231.

23. Fontaine MJ, Shih $H$, Schäfer R, Pittenger MF. Unraveling the mesenchymal stromal cells' paracrine immunomodulatory effects. Transfus Med Rev. 2016; 30(1):37-43.

24. Boregowda SV, Krishnappa V, Phinney DG. Isolation of mouse bone marrow mesenchymal stem cells. Methods Mol Biol. 2016;1416:205-23.

25. Naglich JG, Metherall JE, Russell DW, Eidels L. Expression cloning of a diphtheria toxin receptor: identity with a heparin-binding EGF-like growth factor precursor. Cell. 1992;69(6):1051-61.

26. Iwamoto R, Higashiyama S, Mitamura T, Taniguchi N, Klagsbrun M, Mekada E. Heparin-binding EGF-like growth factor, which acts as the diphtheria toxin receptor, forms a complex with membrane protein DRAP27/CD9, which up-regulates functional receptors and diphtheria toxin sensitivity. EMBO J. 1994;13(10):2322-30.

27. Le Blanc K, Tammik C, Rosendahl K, Zetterberg E, Ringdén O. HLA expression and immunologic properties of differentiated and undifferentiated mesenchymal stem cells. Exp Hematol. 2003;31(10):890-6.

28. Jung DI, Ha J, Kang BT, Kim JW, Quan FS, Lee JH, Woo EJ, Park HM. A comparison of autologous and allogenic bone marrow-derived mesenchymal stem cell transplantation in canine spinal cord injury. J Neurol Sci. 2009;285(1-2):67-77.

29. Pal R, Gopinath C, Rao NM, Banerjee P, Krishnamoorthy V, Venkataramana NK, Totey S. Functional recovery after transplantation of bone marrowderived human mesenchymal stromal cells in a rat model of spinal cord injury. Cytotherapy. 2010;12(6):792-806.

30. Gu W, Zhang F, Xue Q, Ma Z, Lu P, Yu B. Transplantation of bone marrow mesenchymal stem cells reduces lesion volume and induces axonal regrowth of injured spinal cord. Neuropathology. 2010;30(3):205-17.

31. Karaoz E, Kabatas S, Duruksu G, Okcu A, Subasi C, Ay B, Musluman M, Civelek E. Reduction of lesion in injured rat spinal cord and partial functional recovery of motility after bone marrow derived mesenchymal stem cell transplantation. Turk Neurosurg. 2012;22(2):207-17.

32. Willis GR, Fernandez-Gonzalez A, Anastas J, Vitali SH, Liu X, Ericsson M, Kwong A, Mitsialis SA, Kourembanas S. Mesenchymal stromal cell exosomes ameliorate experimental bronchopulmonary dysplasia and restore lung function through macrophage immunomodulation. Am J Respir Crit Care Med. 2018;197(1):104-16

33. Otero-Ortega L, Gómez de Frutos MC, Laso-García F, Rodríguez-Frutos B, Medina-Gutiérrez E, López JA, Vázquez J, Díez-Tejedor E, Gutiérrez-Fernández M. Exosomes promote restoration after an experimental animal model of intracerebral hemorrhage. J Cereb Blood Flow Metab. 2018;38(5):767-79.

34. Nguyen DC, Lewis HC, Joyner C, Warren V, Xiao H, Kissick HT, Wu R, Galipeau J, Lee FE. Extracellular vesicles from bone marrow-derived mesenchymal stromal cells support. J Extracell Vesicles. 2018;7(1):1463778.

35. Farinazzo A, Angiari S, Turano E, Bistaffa E, Dusi S, Ruggieri S, Bonafede R, Mariotti R, Constantin G, Bonetti B. Nanovesicles from adipose-derived mesenchymal stem cells inhibit T lymphocyte trafficking and ameliorate chronic experimental autoimmune encephalomyelitis. Sci Rep. 2018;8(1):7473.
36. Seo M, Kim JC, Kim HK, Choi EW, Jeong S, Nam KC, Jang M. A novel secretory vesicle from deer antlerogenic mesenchymal stem cell-conditioned media (DaMSC-CM) promotes tissue regeneration. Stem Cells Int. 2018;2018:3891404.

37. Mayourian J, Ceholski DK, Gorski PA, Mathiyalagan P, Murphy JF, Salazar SI, Stillitano F, Hare JM, Sahoo S, Hajjar RJ, et al. Exosomal microRNA-21-5p mediates mesenchymal stem cell paracrine effects on human cardiac tissue contractility. Circ Res. 2018;122(7):933-44.

38. Damania A, Jaiman D, Teotia AK, Kumar A. Mesenchymal stromal cellderived exosome-rich fractionated secretome confers a hepatoprotective effect in liver injury. Stem Cell Res Ther. 2018;9(1):31.

39. Ruppert KA, Nguyen TT, Prabhakara KS, Toledano Furman NE, Srivastava AK, Harting MT, Cox CS, Olson SD. Human mesenchymal stromal cell-derived extracellular vesicles modify microglial response and improve clinical outcomes in experimental spinal cord injury. Sci Rep. 2018;8(1):480.

40. Harting MT, Srivastava AK, Zhaorigetu S, Bair H, Prabhakara KS, Toledano Furman NE, Vykoukal JV, Ruppert KA, Cox CS, Olson SD. Inflammationstimulated mesenchymal stromal cell-derived extracellular vesicles attenuate inflammation. Stem Cells. 2018;36(1):79-90.

41. Kim JW, Ha KY, Molon JN, Kim YH. Bone marrow-derived mesenchymal stem cell transplantation for chronic spinal cord injury in rats: comparative study between intralesional and intravenous transplantation. Spine (Phila Pa 1976). 2013;38(17):E1065-74.

42. Kang ES, Ha KY, Kim YH. Fate of transplanted bone marrow derived mesenchymal stem cells following spinal cord injury in rats by transplantation routes. J Korean Med Sci. 2012;27(6):586-93.

43. Cízková D, Rosocha J, Vanický I, Jergová S, Cízek M. Transplants of human mesenchymal stem cells improve functional recovery after spinal cord injury in the rat. Cell Mol Neurobiol. 2006;26(7-8):1167-80.

44. Dominici M, Le Blanc K, Mueller I, Slaper-Cortenbach I, Marini F, Krause D, Deans R, Keating A, Prockop D, Horwitz E. Minimal criteria for defining multipotent mesenchymal stromal cells. The International Society for Cellular Therapy position statement. Cytotherapy. 2006;8(4):315-7.

45. Ahuja CS, Wilson JR, Nori S, Kotter MRN, Druschel C, Curt A, Fehlings MG. Traumatic spinal cord injury. Nat Rev Dis Primers. 2017;3:17018.

46. Choo AM, Liu J, Lam CK, Dvorak M, Tetzlaff W, Oxland TR. Contusion, dislocation, and distraction: primary hemorrhage and membrane permeability in distinct mechanisms of spinal cord injury. J Neurosurg Spine. 2007;6(3):255-66.

47. Renard P, Zachary MD, Bougelet C, Mirault ME, Haegeman G, Remacle J, Raes M. Effects of antioxidant enzyme modulations on interleukin-1-induced nuclear factor kappa B activation. Biochem Pharmacol. 1997;53(2):149-60.

48. Fitzgerald DC, Meade KG, McEvoy AN, Lillis L, Murphy EP, MacHugh DE, Baird AW. Tumour necrosis factor-alpha (TNF-alpha) increases nuclear factor kappaB (NFkappaB) activity in and interleukin-8 (IL-8) release from bovine mammary epithelial cells. Vet Immunol Immunopathol. 2007;116(1-2):59-68.

49. Livolsi A, Busuttil V, Imbert V, Abraham RT, Peyron JF. Tyrosine phosphorylation-dependent activation of NF-kappa B. Requirement for p56 LCK and ZAP-70 protein tyrosine kinases. Eur J Biochem. 2001;268(5):1508-15.

50. Akyurekli C, Le Y, Richardson RB, Fergusson D, Tay J, Allan DS. A systematic review of preclinical studies on the therapeutic potential of mesenchymal stromal cell-derived microvesicles. Stem Cell Rev. 2015;11(1):150-60.

51. Monsel A, Zhu YG, Gennai S, Hao Q, Hu S, Rouby JJ, Rosenzwajg M, Matthay MA, Lee JW. Therapeutic effects of human mesenchymal stem cell-derived microvesicles in severe pneumonia in mice. Am J Respir Crit Care Med. 2015;192(3):324-36. 